\title{
Undoing aggregation
}

Amyloid plaques are characteristic of Alzheimer's disease (AD), but it is unclear how they contribute to the neuropathology. Plaque development involves the assembly of soluble amyloid- $\beta(\mathrm{A} \beta)$ peptides into mature amyloid fibrils through the generation of intermediary protofibrils, which are thought to be the neurotoxic species that contributes to disease progression. This pathway was believed to proceed in only a forward direction, but Martins et al. now show that lipids found in the brain can convert insoluble amyloid fibrils back into soluble protofibrils, providing an additional source of these toxic oligomers.

Recently it has been suggested that altered lipid metabolism might contribute to AD pathology; however, a link between lipid function and amyloid-mediated neurotoxicity has not been established. Here, the authors investigated whether lipids can influence the neurotoxicity of amyloid species on hippocampal cultures. Treatment of the cultures with mature amyloid fibrils alone was not neurotoxic. However, when the fibrils were pre-incubated with liposomes containing synthetic or biological lipids, the mixture induced apoptosis.

To investigate the nature of the toxic mixture in more detail, the authors carried out a series of biophysical assays. They found that the lipids caused the mature amyloid fibrils to revert back to a protofibrillar state. Further analysis revealed that these 'backward protofibrils' had similar morphology and toxicity to protofibrils derived from the assembly of $\mathrm{A} \beta$ monomers.

To determine the effects of the backward protofibrils in vivo, the authors injected the mixture of mature amyloid fibrils and liposomes into the brains of adult mice. They showed that the protofibrils diffused rapidly throughout the brain and caused mild neurotoxicity in the areas in which they concentrated. The protofibrils caused functional effects similar to those that are observed when 'forward protofibrils' are introduced into the brain, including transient impairments in memory formation and fear conditioning, as well as hyperlocomotion and hyperactivity.
These findings suggest that amyloid plaques might not be as stable as was previously assumed: they might provide a source of toxic amyloid protofibrils that can be released by the actions of endogenous brain lipids. This might explain why the number and size of amyloid plaques does not always correspond to the level of functional deficits in patients: a balance between the formation of stable inert amyloid fibrils and destabilization into toxic protofibrils, regulated by the levels of biological lipids present, might be more important.

Katherine Whalley

\section{ORIGINAL RESEARCH PAPER}

Martins, I. C. et al. Lipids revert inert $A \beta$ amyloid fibrils to neurotoxic protofibrils that affect learning in mice. EMBO J. 6 Dec 2007 (doi:10.1038/sj.emboj.7601953)

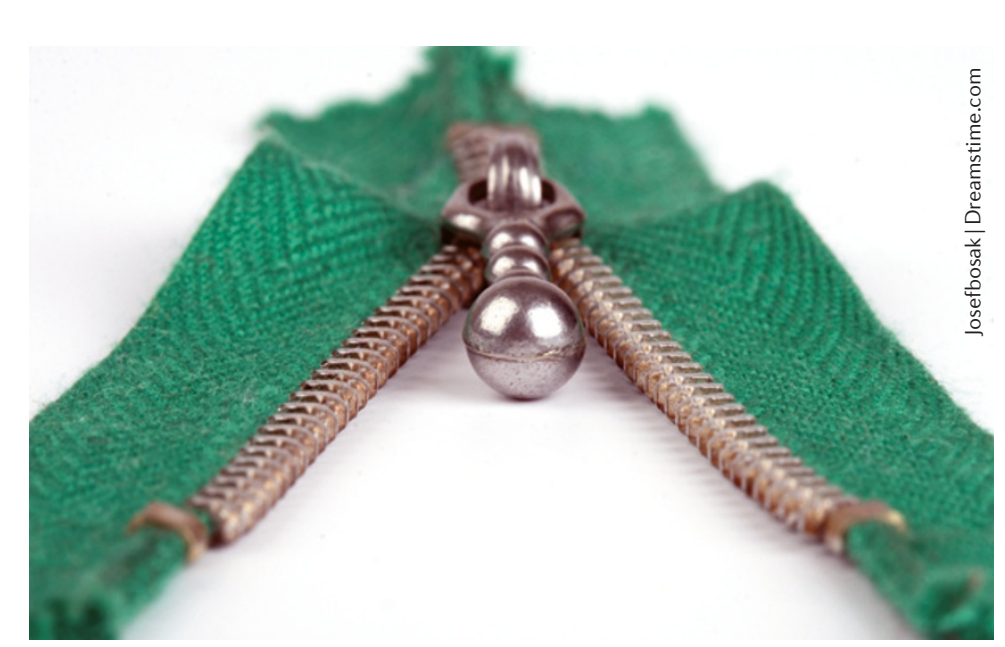

